\title{
Rogue Waves in the Ocean, the Role of Modulational Instability, and Abrupt Changes of Environmental Conditions that can Provoke non Equilibrium Wave Dynamics
}

Karsten Trulsen

Dedicated to Eugene G. Morozov on his 70th Birthday

\begin{abstract}
Modulational instability is an efficient mechanism for the generation of rogue waves in the limit of narrow-banded and long-crested wave fields. While such wave fields are easily achieved in laboratories, there appears to be lacking evidence that known occurrences of rogue waves in the ocean (e.g. Draupner "New Year" wave, Andrea wave) or ship accidents that could have been provoked by rogue waves (e.g. the Prestige accident) actually happened in sea states favorable for the modulational instability to have played an important role. The absence of modulational instability does not mean that nonlinear interactions are unimportant. Here we point out recent results that suggest large deviations from Gaussian statistics can happen due to nonlinearity in the absence of modulational instability, the key ingredient seems to be that the wave field is brought into a state of non-equilibrium.
\end{abstract}

\section{Introduction}

Rogue or freak waves are waves that are unexpectedly large in comparison with ambient waves. The term "freak wave" was apparently introduced by Draper (1964) who discussed ship accidents that could have been provoked by such waves. Mallory (1974) discussed serious consequences of extreme waves on ship traffic in the Agulhas current off South Africa. With the advent of offshore oil and gas exploration, well documented observations of freak waves accumulated (e.g. Sand et al, 1990). The "New Year" wave at Draupner in the North Sea 1/1-1995 is one of the best documented observations of a rogue wave (Haver, 2004; Cavaleri et al, 2016). Later the even more extreme Andrea wave at Ekofisk in the North Sea 9/11-2007

Karsten Trulsen

Department of Mathematics, University of Oslo, Norway, e-mail: karstent@math.uio.no 
was also well documented (Magnusson and Donelan, 2013; Bitner-Gregersen et al, 2014; Donelan and Magnusson, 2017).

This is a good opportunity to give praise to the policy of openness demonstrated by the Norwegian oil company Statoil giving academic researchers unrestricted access to the Draupner "New Year" wave dataset. Few measured time series have been published more often, or inspired more wave research, than the one shown in figure 1.

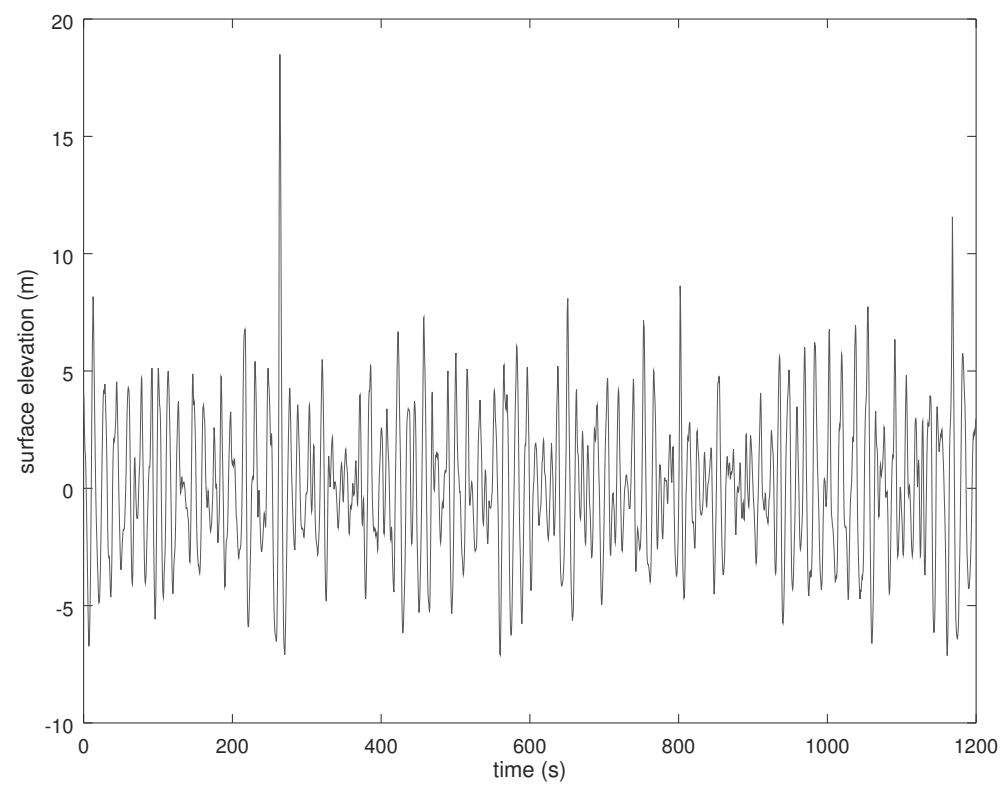

Fig. 1 Twenty minute wave elevation time series measured by a downward pointing radar at 16/11E in the Norwegian sector of the North Sea on 1/1-1995. First axis is time in seconds, second axis is elevation in meters. Data courtesy of J. I. Dalane and O. T. Gudmestad of Statoil.

There is currently no consensus on how to define rogue or freak waves. Common criteria are $H / H_{s}>2$ or $\eta_{c} / H_{s}>1.25$ where $H$ is the zero-crossing wave height, $\eta_{c}$ is the crest height, and $H_{s}$ is the significant wave height defined as four times the standard deviation of the surface elevation typically calculated from a 20 minute time series (Haver, 2000; Dysthe et al, 2008).

The existence of rogue waves in the ocean is nowadays well accepted and they are recognized as a threat and a challenge for human activity offshore (Dysthe et al, 2008; Kharif et al, 2009; Bitner-Gregersen and Gramstad, 2016). Indeed, recently a large wave killed one person and injured several others in the COSL Innovator accident at Troll in the Norwegian Sea 30/12-2015 (Viste-Ollestad et al, 2016). 
Most attention to rogue wave generation has so far been given to random events within Gaussian seas, including linear refraction due to currents or bathymetries, random events within slightly non-Gaussian seas due to static nonlinearities or dynamic nonlinear evolution in equilibrium wave fields, and modulational instabilities which are nonlinear instabilities of perturbations around steady states. While such mechanisms can indeed produce freak waves, and such criteria are nowadays used as warning criteria in operational forecasting, validation performed by e.g. the Norwegian Meteorological Institute has shown that some improvements are still necessary for the warning criteria to be fully satisfactory (Bitner-Gregersen and Gramstad, 2016).

This paper points out a possible future direction for rogue wave research that might provide some of the desired improvements. There is indeed a different path to rogue wave formation, one that has received little attention so far and is not accounted for in the above practices and criteria, namely the dynamic evolution of non-equilibrium wave fields, where the lack of equilibrium is not due to a small perturbation away from a steady state.

Recent laboratory experiments (Trulsen et al, 2012; Raustøl, 2014) and numerical computations (Gramstad et al, 2013; Viotti and Dias, 2014) have shown that non-equilibrium evolution of wave fields can produce surprisingly rough wave conditions. We anticipate that wave fields that are brought out of equilibrium due to rapidly varying meteorological conditions, or significantly non-uniform environments such as currents or bathymetries (Onorato and Suret, 2016), or possibly the sudden appearance of a ship in a wave field (Molin et al, 2005, 2014), can be a significant source of rogue waves.

The paper is organized as follows: $\S 2$ gives a summary of common theories on which current rogue wave warning criteria are typically based, $\S 3$ presents a case study for the involvement of rogue waves in the Prestige oil tanker accident based on the common theories, $\S 4$ points out an alternative type of mechanism for rogue wave generation typically not included in todays warning criteria, and $\S 5$ provides a conclusion.

\section{Common Theories for Rogue Waves}

Within linear wave theory (LWT) there are several mechanisms that can provoke large waves, e.g. spatio-temporal focusing of waves, refraction over uneven depth and refraction over non-uniform currents. Within LWT, employing the principle of superposition and the Central Limit Theorem from probability theory, it is anticipated that the resulting distribution of surface elevation is Gaussian (e.g. Pierson, 1955).

Rogue waves are known to occur more often than anticipated from LWT, this enhanced occurrence is generally accepted to be due to nonlinearity. There are several known nonlinear mechanisms that can be responsible for this. 
Second-order corrections to deep-water waves, static or bound wave nonlinear corrections in general, are known to provoke small deviation from Gaussian statistics. These corrections were derived for uniform waves by Stokes (1847), for deepwater irregular gravity waves by Tick (1959) and Longuet-Higgins (1963) and further by Masuda et al (1979). Static nonlinear corrections to linear wave theory form the basis for Tayfun-distributions (Tayfun, 1980).

Starting with the observation that steady uniform waves are unstable to small perturbations, the Benjamin-Feir instability (Benjamin, 1967; Benjamin and Feir, 1967), or more general modulational instability (MI) was soon recognized as a mechanism that could initiate the generation of extreme waves. It was soon recognized that the cubic nonlinear Schrödinger (NLS) equation (Zakharov, 1968; Benney and Roskes, 1969; Davey, 1972; Hasimoto and Ono, 1972) is the simplest nonlinear model that accounts for this instability mechanism. The MI occurs if the ratio between the steepness of the uniform wave and the spectral bandwidth of the perturbation is above a threshold.

Soon after the first well-documented observations of rogue waves in the ocean, e.g. the Draupner wave, it was suggested that the generation of such waves in the ocean could be explained by weakly nonlinear and narrow-banded models, in particular by the nonlinear Schrödinger (NLS) equation. Trulsen and Dysthe (1997) argued that it would be an advantage to use a broader-bandwidth modification of the modified nonlinear Schrödinger (MNLS) equation of Dysthe (1979) for better representation of realistic bandwidths (Trulsen and Dysthe, 1996; Trulsen et al, 2000).

Breather solutions (Kuznetsov, 1977; Ma, 1979; Peregrine, 1983; Dysthe and Trulsen, 1999; Osborne et al, 2000) are long-time and long-distance solutions of the NLS equation, starting from an infinitesimal perturbation of a uniform wave train, resulting in extreme waves localized in space and time, occurring once or repeatedly in space and/or in time. Breather solutions have recently been studied in laboratory experiments (Chabchoub et al, 2011, 2012, 2014).

Related to the discovery of modulational instability of uniform wave trains, it was also recognized that steady homogeneous wave fields are unstable to small inhomogeneous perturbations (Alber and Saffman, 1978; Alber, 1978; Crawford et al, 1980). This instability occurs if the ratio between the steepness and the spectral bandwidth of the wave field is above a threshold.

The Benjamin-Feir index (BFI), coined by Janssen (2003), previously suggested by Onorato et al $(2001,2002 b)$ under a different name, is precisely the ratio between the steepness and the spectral bandwidth of the wave field. It has been suggested as an indicator to predict increased probability of rogue waves. The BFI has been shown to be useful as an indicator for extreme waves in unidirectional seas (Onorato et al, 2004, 2006) easily reproduced in long and narrow laboratory tanks. However, the BFI has been found to be less useful in directional seas in simulations (Onorato et al, 2002a; Gramstad and Trulsen, 2007) and in laboratory tests (Onorato et al, 2009b,a) as well as an operational forecasting criterion (Bitner-Gregersen and Gramstad, 2016).

A different path from swell and wind-sea interaction to freak wave generation was suggested by Tamura et al (2009) who speculated that the nonlinear coupling 
between swell and wind-sea could generate a narrow spectrum. Some ship accidents indeed seem to have occurred in conditions of narrowing wave spectra (Tamura et al, 2009; Waseda et al, 2012, 2014). It has indeed been observed that the directional spread sometimes have been reduced ahead of increased rogue wave occurrences at sea (Toffoli and Bitner-Gregersen, 2011).

Recently, researchers have started suspecting that MI is not the correct or the only path to explain real world ocean rogue waves, the waves being in general too broad-banded and short-crested for the BFI to be useful (e.g. Fedele et al, 2016).

\section{Case Study on Rogue Waves Through Common Theories: The Prestige Accident}

Freak waves are sometimes the subject of great controversies. There is for example still no consensus on the cause of the Prestige oil tanker initial accident on 13/112002 , which subsequently led to a major environmental disaster after the sinking of the ship on 19/11-2002. The magnitude of the environmental disaster provoked heated debate in mass media and in court regarding the likelihood that the initial accident could have been caused by a rogue wave (see Trulsen et al, 2015).

In the recent study of Trulsen et al (2015) newly computed hindcast spectra for every hour during the day of the accident were used as input data for four different nonlinear models capable of computing the phase-resolved sea surface, allowing to estimate statistical parameters that characterize the conditions for rogue waves. All four models coincided that the wave conditions encountered by the tanker Prestige at the moment of the accident were slightly more extreme than those of a Gaussian sea state, and slightly less extreme than those of a Tayfun sea state. This study strongly suggests that the probability of a rogue wave hitting the oil tanker was neither greater than nor smaller than usual.

\section{Rogue Waves in non Equilibrium Wave Fields}

Practical experience with the common theories for rogue waves, e.g. application of the BFI as a warning criterion, suggests that some improvements may still be necessary for the warning criteria to be fully satisfactory (Bitner-Gregersen and Gramstad, 2016). We here point out that there is indeed another path to the formation of rogue waves.

It is often observed that numerical simulation of nonlinear wave evolution, initialized with artificial initial conditions, can need some evolution time or distance before the wave fields become well-behaved. During the initial transient evolution extreme events are often seen to occur. Some effort has been made to suppress this behavior, e.g. by Dommermuth (2000), although the "problem" is typically dealt with simply letting the numerical simulations run over sufficient time or distance 
before the results are used. On the other hand, a very interesting situation would arise if this "initial" strange behavior was the result of a sudden change of physical environment rather than artificial initialization of a numerical integration.

Recently it has been observed that irregular wave fields that propagate from deeper waters into shallower waters can have significant amplification of kurtosis and freak wave statistics some distance inside the transition to the shallower depth. This behavior was first discovered in an experimental dataset from MARIN in The Netherlands by Trulsen et al (2012), subsequently is was studied numerically by Sergeeva et al (2011), Zeng and Trulsen (2012), Gramstad et al (2013) and Viotti and Dias (2014). This is a nonlinear effect that is neither explained by MI nor by linear refraction.

Recently Raustøl (2014) carried out fine-resolution experiments and measured that the kurtosis could be amplified to a value of 6, occurring at a location approximately one wavelength on the inside of the depth transition to shallower water. She also identified thresholds for water depths when this amplifying behavior takes place. It is interesting to note that this extreme amplification of kurtosis took place precisely in a wave field that was not modulationally unstable.

It is common to treat a sea state as being statistically stationary when in fact it varies. Meteorological forecasting services typically give forecasts for every three hours. In Trulsen et al (2015) the Prestige accident was studied with hindcasts every hour, making the assumption that the sea state was constant during each of the onehour intervals. In the case that the sea state varied dramatically within the one-hour intervals, the nonlinear phase-resolving simulations of Trulsen et al (2015) could be rendered invalid. An insufficient amount of work has been done to identify what happens if the meteorological conditions and sea state change sufficiently fast that the wave field is not in an equilibrium state. Indeed, Tamura et al (2009) suggested that sea states can vary in time in such a way that the occurrence of rogue waves may be affected.

The presence of a ship in a wave field is known to locally affect the wave field near the ship. If the ship appears suddenly into an already established wave field, it may also represent a perturbation that brings the wave field temporarily out of equilibrium (Molin et al, 2005, 2014).

Indeed, in the recent review of Onorato and Suret (2016) it is speculated that a change of ambient conditions can bring a wave field out of equilibrium, thus provoking amplification of kurtosis before the wave field is brought back to equilibrium.

\section{Conclusions}

Rogue waves are known to occur more frequently than expected from linear wave theory, more frequently than expected within Gaussian seas. The common nonlinear theories for rogue waves explain such deviation by weakly nonlinear effects on top of equilibrium linear sea states, or as the effect of modulational instability due to unstable perturbations of steady states. The degree of modulational instability of a 
steady sea state is sometimes assessed by the so-called Benjamin-Feir Index (BFI). There is however a different mechanism for rogue wave generation, viz. nonlinear dynamics of wave fields that are not in an equilibrium state. This mechanism is not indicated by the value of BFI, since the modulational instability is not relevant in the absence of a steady state. We have recently performed experiments at the Department of Mathematics at the University of Oslo revealing that a substantial amplification of kurtosis can occur in non-equilibrium wave fields that are not modulationally unstable.

This work has been supported by the Research Council of Norway through the project "EXtreme wave WArning criteria for MARine structures" (ExWaMar) RCN 256466.

\section{References}

Alber IE (1978) The effects of randomness on the stability of two-dimensional surface wavetrains. Proc R Soc Lond A 363:525-546

Alber IE, Saffman P (1978) Stability of random nonlinear deep water waves with finite bandwidth spectra. Tech. Rep. 31326-6035-RU-, TRW Defense and Space System Group

Benjamin TB (1967) Instability of periodic wavetrains in nonlinear dispersive systems. Proc R Soc Lond A 299:59-75

Benjamin TB, Feir JE (1967) The disintegration of wave trains on deep water. J Fluid Mech 27:417-430

Benney DJ, Roskes GJ (1969) Wave instabilities. Studies Appl Math 48:377-385

Bitner-Gregersen EM, Gramstad O (2016) Rogue waves - Impact on ships and offshore structures. Tech. Rep. 05-2015, DNV-GL

Bitner-Gregersen EM, Fernandez L, Lefèvre JM, Monbaliu J, Toffoli A (2014) The North Sea Andrea storm and numerical simulations. Nat Hazards Earth Syst Sci 14:1407-1415

Cavaleri L, Barbariol F, Benetazzo A, Bertotti L, Bidlot JR, Janssen P, Wedi N (2016) The Draupner wave: A fresh look and the emerging view. J Geophys Res Oceans 121:6061-6075

Chabchoub A, Hoffmann NP, Akhmediev N (2011) Rogue wave observation in a water wave tank. Phys Rev Lett 106:204,502

Chabchoub A, Akhmediev N, Hoffmann NP (2012) Experimental study of spatiotemporally localized surface gravity water waves. Phys Rev E 86:016,311

Chabchoub A, Kibler B, Dudley JM, Akhmediev N (2014) Hydrodynamics of periodic breathers. Phil Trans R Soc Lond A 372:20140,005

Crawford DR, Saffman PG, Yuen HC (1980) Evolution of a random inhomogeneous field of nonlinear deep-water gravity waves. Wave Motion 2:1-16

Davey A (1972) The propagation of a weak nonlinear wave. J Fluid Mech 53:769781 
Dommermuth D (2000) The initialization of nonlinear waves using an adjustment scheme. Wave Motion 32:307-317

Donelan MA, Magnusson AK (2017) The making of the Andrea wave and other rogues. Scientific Reports 7:44,124

Draper L (1964) 'Freak' ocean waves. Oceanus 10:13-15

Dysthe K, Krogstad HE, Müller P (2008) Oceanic rogue waves. Annu Rev Fluid Mech 40:287-310

Dysthe KB (1979) Note on a modification to the nonlinear Schrödinger equation for application to deep water waves. Proc R Soc Lond A 369:105-114

Dysthe KB, Trulsen K (1999) Note on breather type solutions of the NLS as models for freak-waves. Physica Scripta T82:48-52

Fedele F, Brennan J, Ponce de León S, Dudley J, Dias F (2016) Real world ocean rogue waves explained without the modulational instability. Scientific Reports $6: 27,715$

Gramstad O, Trulsen K (2007) Influence of crest and group length on the occurrence of freak waves. J Fluid Mech 582:463-472

Gramstad O, Zeng H, Trulsen K, Pedersen GK (2013) Freak waves in weakly nonlinear unidirectional wave trains over a sloping bottom in shallow water. Phys Fluids 25, 122103

Hasimoto H, Ono H (1972) Nonlinear modulation of gravity waves. J Phys Soc Japan 33:805-811

Haver S (2000) Evidences of the existence of freak waves. In: Rogue Waves 2000, Ifremer, pp 129-140

Haver S (2004) A possible freak wave event measured at the Draupner jacket Januar 11995. http://www.ifremer.fr/web-com/stw2004/rw/fullpapers/walk_on_haver.pdf. In: Rogue Waves 2004, pp 1-8

Janssen PAEM (2003) Nonlinear four-wave interactions and freak waves. J Phys Oceanogr 33:863-884

Kharif C, Pelinovsky E, Slunyaev A (2009) Rogue waves in the ocean. Springer

Kuznetsov EA (1977) Solitons in a parametrically unstable plasma. Sov Phys Dokl 22:507-508

Longuet-Higgins MS (1963) The effect of non-linearities on statistical distributions in the theory of sea waves. J Fluid Mech 17:459-480

Ma YC (1979) The perturbed plane-wave solutions of the cubic Schrödinger equation. Studies Appl Math 60:43-58

Magnusson AK, Donelan MA (2013) The Andrea wave characteristics of a measured North Sea rogue wave. JOMAE 135:031,108

Mallory JK (1974) Abnormal waves on the south east coast of South Africa. Int Hydrog Rev 51:99-129

Masuda A, Kuo YY, Mitsuyasu H (1979) On the dispersion relation of random gravity waves. Part 1. Theoretical framework. J Fluid Mech 92:717-730

Molin B, Remy F, Kimmoun O, Jamois E (2005) The role of tertiary wave interactions in wave-body problems. J Fluid Mech 528:323-354 
Molin B, Kimmoun O, Remy F, Chatjigeorgiou IK (2014) Third-order effects in wave-body interaction. Eur J Mech B/Fluids 47:132-144

Onorato M, Suret P (2016) Twenty years of progresses in oceanic rogue waves: the role played by weakly nonlinear models. Natural Hazards 84:541-548

Onorato M, Osborne AR, Serio M, Bertone S (2001) Freak waves in random oceanic sea states. Phys Rev Lett 86:5831-5834

Onorato M, Osborne AR, Serio M (2002a) Extreme wave events in directional, random oceanic sea states. Phys Fluids 14:L25-L28

Onorato M, Osborne AR, Serio M, Resio D, Pushkarev A, Zakharov VE, Brandini C (2002b) Freely decaying weak turbulence for sea surface gravity waves. Phys Rev Lett 89:144,501-1-144,501-4

Onorato M, Osborne AR, Serio M, Cavaleri L, Brandini C, Stansberg CT (2004) Observation of strongly non-Gaussian statistics for random sea surface gravity waves in wave flume experiments. Phys Rev E 70:1-4

Onorato M, Osborne AR, Serio M, Cavaleri L, Brandini C, Stansberg CT (2006) Extreme waves, modulational instability and second order theory: wave flume experiments on irregular waves. Eur J Mech B/Fluids 25:586-601

Onorato M, Cavaleri L, Fouques S, Gramstad O, Janssen PAEM, Monbaliu J, Osborne AR, Pakozdi C, Serio M, Stansberg CT, Toffoli A, Trulsen K (2009a) Statistical properties of mechanically generated surface gravity waves: A laboratory experiment in a three dimensional wave basin. J Fluid Mech 627:235-257

Onorato M, Waseda T, Toffoli A, Cavaleri L, Gramstad O, Janssen PAEM, Kinoshita T, Monbaliu J, Mori N, Osborne AR, Serio M, Stansberg CT, Tamura H, Trulsen K (2009b) Statistical properties of directional ocean waves: The role of the modulational instability in the formation of extreme events. Phys Rev Lett 102:114,502

Osborne AR, Onorato M, Serio M (2000) The nonlinear dynamics of rogue waves and holes in deep-water gravity wave trains. Phys Lett A 275:386-393

Peregrine DH (1983) Water-waves, non-linear Schrödinger-equations and their solutions. J Austral Math Soc B 25:16-43

Pierson WJ (1955) Wind generated gravity waves. Advances in Geophysics 2:93178

Raustøl A (2014) Freake bølger over variabelt dyp. Master’s thesis, University of Oslo

Sand SE, Ottesen Hansen NE, Klinting P, Gudmestad OT, Sterndorff MJ (1990) Freak wave kinematics. In: Tørum A, Gudmestad OT (eds) Water Wave Kinematics, Kluwer, pp 535-549

Sergeeva A, Pelinovsky E, Talipova T (2011) Nonlinear random wave field in shallow water: variable Korteweg-de Vries framework. Nat Hazards Earth Syst Sci $11: 323-330$

Stokes GG (1847) On the theory of oscillatory waves. Trans Camb Philos Soc 8:441-455

Tamura H, Waseda T, Miyazawa Y (2009) Freakish sea state and swell-windsea coupling: Numerical study of the Suwa-Maru incident. Geophys Res Lett 36:L01,607

Tayfun MA (1980) Narrow-band nonlinear sea waves. J Geophys Res 85:1548-1552 
Tick LJ (1959) A non-linear random model of gravity waves I. J Math Mech 8:643651

Toffoli A, Bitner-Gregersen EM (2011) Extreme and rogue waves in directional wave fields. Open Ocean Engineering Journal 4:24-33

Trulsen K, Dysthe KB (1996) A modified nonlinear Schrödinger equation for broader bandwidth gravity waves on deep water. Wave Motion 24:281-289

Trulsen K, Dysthe KB (1997) Freak waves - a three-dimensional wave simulation. In: Proceedings of the 21st Symposium on Naval Hydrodynamics, National Academy Press, pp 550-560

Trulsen K, Kliakhandler I, Dysthe KB, Velarde MG (2000) On weakly nonlinear modulation of waves on deep water. Phys Fluids 12:2432-2437

Trulsen K, Zeng H, Gramstad O (2012) Laboratory evidence of freak waves provoked by non-uniform bathymetry. Phys Fluids 24:097,101

Trulsen K, Nieto Borge JC, Gramstad O, Aouf L, Lefèvre JM (2015) Crossing sea state and rogue wave probability during the Prestige accident. J Geophys Res Oceans 120:7113-7136

Viotti C, Dias F (2014) Extreme waves induced by strong depth transitions: Fully nonlinear results. Phys Fluids 26:051,705

Viste-Ollestad I, Andersen TL, Oma N, Zachariassen S (2016) Granskingsrapport etter hendelse med fatalt utfall på COSL Innovator 30. desember 2015. Tech. rep., Petroleumstilsynet

Waseda T, Tamura H, Kinoshita T (2012) Freakish sea index and sea states during ship accidents. J Mar Sci Technol 17:305-314

Waseda T, In K, Kiyomatsu K, Tamura H, Miyazawa Y, Iyama K (2014) Predicting freakish sea state with an operational third-generation wave model. Nat Hazards Earth Syst Sci 14:945-957

Zakharov VE (1968) Stability of periodic waves of finite amplitude on the surface of a deep fluid. J Appl Mech Tech Phys 9:190-194

Zeng H, Trulsen K (2012) Evolution of skewness and kurtosis of weakly nonlinear unidirectional waves over a sloping bottom. Nat Hazards Earth Syst Sci 12:631638 